\title{
Cardiovascular Comorbidities in Women with Psoriasis
}

\author{
Durga Prasad Annabathula ${ }^{1}$ \\ ${ }^{1}$ Department of Dermatology, DERMACARE, Hyderabad, India \\ Ind J Car Dis Wom:2020;5:109-110
}

The prevalence of cardiovascular (CV) risk factors among psoriasis patients is well-known, and therefore there is an elevated risk of CV disease (CVD). Psoriasis is associated with an elevated risk of myocardial infarction, stroke, and also death caused by CVD, which are as a whole referred to as major adverse cardiovascular events (MACE). ${ }^{1}$ It also has been detailed that the magnitude of these risk factors is directly proportionate to the severity of psoriasis. ${ }^{1}$

Atherosclerosis, which is a chronic inflammatory disease of the blood vessels, is usually the cause for CVD. Atherosclerosis is associated with both innate and adaptive immune responses that involve the Th- 1 and Th-17 pathways., ${ }^{2,3}$ On the other hand, psoriasis is also a chronic inflammatory disorder which displays its association with Th- 1 and Th- 17 mediated inflammation, resulting in production of IL-17, INF-gamma and TNF and leading to the formation of plaques over the skin. This similarity in the immunological and inflammatory pathways implies a relationship between CVD and psoriasis. ${ }^{3,4}$

The various determinants of CVD evaluated in this study ${ }^{5}$ were elevated waist circumference $(>80 \mathrm{~cm})$, raised triglycerides ( $>150 \mathrm{mg} / \mathrm{dL}$ ), reduced high-density lipoprotein $(\mathrm{HDL})(<50)$, fasting blood glucose $(>100 \mathrm{mg} / \mathrm{dL})$, and raised blood pressure which constitute the metabolic syndrome, high-sensitivity C-reactive protein (hsCRP), weight and carotid intima media thickness (CIMT). The various other variables (related to psoriasis) that were recorded in this study ${ }^{5}$ are the duration of psoriasis, family history of the same, intake of drugs that trigger psoriasis, and the severity of the disease which was assessed by Psoriasis Area Severity Index (PASI) and body surface area (BSA). The other quality scoring systems of psoriasis that are methodologically validated are PGA (Physician's Global Assessment)/IGA mod 2011 (Investigator's Global Assessment modified in 2011), LS-PGA (Lattice System-Physician's Global Assessment, and SPI (Salford psoriasis index). ${ }^{6}$ These assessment tools have, however, not been used in this study ${ }^{5}$ to assess the severity of the disease which can be considered as a demerit of the study. ${ }^{5}$
The incidence of metabolic syndrome in women was found to be higher than in men, owing to the preponderance of women to develop central obesity (increased waist circumference). ${ }^{7}$ Also, inflammation increases the degree of occurrence of metabolic syndrome, particularly in women, which, in turn, elevates the CV risk. ${ }^{8}$ Given that psoriasis is a chronic inflammatory disease, it poses a greater risk of CVD in women. ${ }^{9}$ This study ${ }^{5}$ showed higher prevalence of metabolic syndrome in cases with psoriasis than in controls, which is in accordance with the research conducted by Regitz-Zagrosek et al.,

It has been concluded in a study done by Pietrzak et al ${ }^{10}$ that the relation of CVD and psoriasis in females intensifies with age. However, no such associations have been recorded in this study. ${ }^{5}$

It is demonstrated by this study ${ }^{5}$ that hsCRP, which is an important indicator of atherosclerosis, was raised in patients with psoriasis, in close agreement with the study carried out by Jindal et al. ${ }^{4}$ It was also stated by this study ${ }^{5}$ that CIMT, which is a salient tool for the measurement of subclinical atherosclerotic changes, was elevated among psoriasis cases than in controls which is, again, in conformity with Jindal et al. ${ }^{4}$

All the other determinants of CVD evaluated in this study ${ }^{5}$ had higher incidence in women with psoriasis than in women without psoriasis, which is in correspondence with Jindal et al. ${ }^{4}$

Therefore, it is of utmost importance that the female patients with psoriasis be orderly screened for the various $\mathrm{CV}$ risk factors for early detection and prevention of CVD.

Conflict of Interest

None declared.

\section{References}

1 Takeshita J, Grewal S, Langan SM, et al. Psoriasis and comorbid diseases: Epidemiology. J Am Acad Dermatol 2017;76(3):377-390

2 Hansson GK, Libby P. The immune response in atherosclerosis: a double-edged sword. Nat Rev Immunol 2006;6(7):508-519

3 Yiu KH, Yeung CK, Zhao CT, et al. Prevalence and extent of subclinical atherosclerosis in patients with psoriasis. J Intern Med 2013;273(3):273-282
Address for correspondence

Durga Prasad Annabathula, MBBS, DD, Department of Dermatology, DERMACARE, Hyderabad, 500047, India (e-mail: dp@dermacareindia.com).
DOI https://doi.org/ 10.1055/s-0040-1713949.

(C)2020 Women in Cardiology and Related Sciences published online

July 9,2020 
4 Jindal S, Jindal N. Psoriasis and cardiovascular diseases: a literature review to determine the causal relationship. Cureus 2018;10(2):e2195

5 BalaS, Mavoori A, Pandve H, et al. Determinants of cardiovascular disease among urban women with psoriasis: a case-control study. Ind J Car Dis Wom 2020; doi: 10.1055/s-0040-1705661

6 Puzenat E, Bronsard V, Prey S, et al. What are the best outcome measures for assessing plaque psoriasis severity? A systematic review of the literature. J Eur Acad Dermatol Venereol 2010;24(Suppl 2):10-16

7 Beigh SH, Jain S. Prevalence of metabolic syndrome and gender differences. Bioinformation 2012;8(13):613-616
8 Regitz-Zagrosek V, Lehmkuhl E, Weickert MO. Gender differences in the metabolic syndrome and their role for cardiovascular disease. Clin Res Cardiol 2006;95(3):136-147

9 Misitzis A, Cunha PR, Kroumpouzos G. Skin disease related to metabolic syndrome in women. Int J Womens Dermatol 2019;5(4):205-212

10 Pietrzak A, Czuczwar P, Mosiewicz J, et al. Cardiovascular disease in psoriatic post-menopausal women. J Eur Acad Dermatol Venereol 2015;29(6):1231-1234 